\title{
LE MARCHÉ DE L'EAU AMÉRICAIN
}

Jean-Michel Brault, Christelle Pezon

Métropolis | « Flux »

2002/1 n 47 | pages 69 à 79

ISSN 1154-2721

Article disponible en ligne à l'adresse :

http://www.cairn.info/revue-flux-2002-1-page-69.htm

\section{!Pour citer cet article :}

Jean-Michel Brault, Christelle Pezon, «Le marché de l'eau américain », Flux 2002/1 (n 47), p. 69-79.

Distribution électronique Cairn.info pour Métropolis.

(C) Métropolis. Tous droits réservés pour tous pays.

La reproduction ou représentation de cet article, notamment par photocopie, n'est autorisée que dans les limites des conditions générales d'utilisation du site ou, le cas échéant, des conditions générales de la licence souscrite par votre établissement. Toute autre reproduction ou représentation, en tout ou partie, sous quelque forme et de quelque manière que ce soit, est interdite sauf accord préalable et écrit de l'éditeur, en dehors des cas prévus par la législation en vigueur en France. Il est précisé que son stockage dans une base de données est également interdit. 


\title{
Le marché de l'eau américain
}

\author{
Entretien avec Jean-Michel Brault
}

par Christelle Pezon

Jean-Michel Brault, ingénieur Arts et Métiers, a occupé différentes fonctions au sein du groupe GTM, de 1973 à 1994, avant de rejoindre la Direction internationale de l'Eau de Lyonnaise des Eaux, aujourd'hui Suez, comme Directeur Amérique du Nord.

En 1997, il est nommé Président Directeur Général des filiales nord-américaines du pôle Eau de Suez, United Water, basé aux USA. Il a rejoint, début 2002, le siège parisien d'ONDEO, le pôle Eau de Suez, à la Direction du Développement. II est également Directeur Général d'ONDEO North America.

Pendant son séjour aux USA, Jean-Michel Brault était membre des comités exécutifs des associations professionnelles des sociétés de services d'eau et représentant du groupe Suez auprès de I'US Chamber of Commerce.

$\bigcup$ nited Water est devenu un acteur majeur de l'alimentation et du traitement de l'eau aux États-Unis, en prenant position sur le marché des services régulés (franchise) et sur le marché des services aux collectivités (contract-out). Propriétaire de réseaux dans le premier cas, UW est régulé, dans chaque État, par une commission de régulation des services publics, responsable du contrôle de l'ensemble des entreprises de service public (énergie, télécommunication, transport, eau et assainissement). Les services aux collectivités consistent à prendre la responsabilité d'une activité (production d'eau potable ou épuration des eaux usées, le plus souvent, mais aussi distribution d'eau potable et collecte d'eaux usées), à travers des contrats
Operation \& Maintenance (O\&M) ou Operation, Maintenance \& Management $(\mathrm{OM} \& M)$, de cinq à vingt ans, en contrepartie d'une rémunération annuelle versée par la ville.

Jean-Michel Brault analyse pour nous le marché de l'eau américain et les atouts de United Water pour s'y développer.

Flux. À travers United Water, Ondeo est devenu un acteur majeur du marché de l'eau américain. D'opérateur propriétaire de réseaux locaux, régulé par les commissions de régulation des services publics (Public Utilities Commission), United Water s'est affirmé comme partenaire contractuel de quelques grandes villes. Comment s'est opérée cette évolution? 
Jean-Michel Brault. Aujourd'hui, il y a 58000 réseaux de distribution d'eau potable dans tout le pays, dont 24000 sont gérés par des opérateurs propriétaires qui desservent 33 millions d'habitants, essentiellement dans des villes moyennes. Jusqu'au début des années 1990, les sociétés américaines étaient propriétaires de leurs actifs et restaient sur leur marché régulé. Aucune n'a tenté de développer des services. De grosses sociétés, notamment d'ingénierie, qui travaillaient pour les collectivités et proposaient des services autour de leur métier de base, ont commencé à développer des contrats de construction clés en main. Puis elles ont proposé des services autour de la gestion et de la maintenance des installations. Ce mouvement a commencé, il y a une petite trentaine d'années, mais il est longtemps resté très marginal. En effet, l'essentiel des investissements réalisés par les collectivités était financé par des crédits fédéraux en franchise d'impôts, qui limitaient à trois ou cinq ans la durée pour laquelle une municipalité pouvait contracter un service à un opérateur privé, sauf à perdre le bénéfice de cette franchise, c'est-à-dire à la rembourser à l'État. Le marché était donc limité. L'Environmental Protection Agency (EPA) I'a réouvert, en 1992, quand elle a pris conscience des besoins d'investissement énormes du secteur eau et assainissement. Des projets pilotes ont été lancés pour essayer d'associer le secteur privé au développement, à travers des Public-Private Partnerships, ce fut notamment un projet de BOT à Petaluma en Californie mais la municipalité n'avait pas de problème de financement. Elle n'était donc pas très motivée pour le réaliser. Elle a joué avec le concept, mais il ne s'est finalement rien passé. Le vrai déclencheur a été Indianapolis. C'était le bas de l'échelle en terme de service puisqu'il s'agissait d'exploitation et de maintenance sans investissement, mais le secteur privé a pu démontrer qu'il avait des compétences techniques et que sa gestion pouvait permettre à une ville importante de faire des économies. Le maire d'Indianapolis, Steve Goldsmith, croyait beaucoup au partenariat public/privé. II avait la volonté de réveiller sa ville dont les habitants désertaient le centre pour la périphérie et d'attirer les investisseurs.

Le marché a alors commencé à bouger. Les opérateurs se sont organisés. Les maires se sont un peu intéressés au concept de la délégation. Une action a été conduite auprès du gouvernement fédéral pour changer les règles de l'administration fis- cale et faire sauter cette barrière de cinq ans. En 1997, la règle a été changée pour aller à vingt ans. Les contrats avec Milwaukee et Atlanta ont suivi.

Depuis les inflexions de 1992 et 1997, on projette une nouvelle accélération au cours des années 2000-2005. Combien de temps faudra-t-il ? personne ne le sait, mais toute l'industrie de I'eau, qu'elle soit publique ou privée, travaille à changer les règles d'accès aux crédits fédéraux en franchise d'impôt. Nous avons deux axes de travail en cours à Washington : les municipal tax rebond et les private activity bond. Les premiers sont donnés aux municipalités pour financer les infrastructures (l'eau et les transports), l'éducation, la sécurité et la santé. Les seconds sont accessibles aux opérateurs privés pour un projet de service public. Ils sont $0,5 \%$ plus chers que les premiers et, surtout, leur montant est plafonné. Le premier effort est de faire sauter ce « cap ». Le deuxième effort est d'obtenir que ces fonds soient donnés directement à l'opérateur privé qui est en situation d'avoir un contrat, plutôt que de les verser à la municipalité. L'avantage pour la municipalité est de faire sortir la dette de son bilan. Pour l'opérateur privé, l'avantage est d'obtenir la gestion des actifs grâce au financement. On s'oriente alors vers un affermage et éventuellement après vers une concession.

Flux. Quels sont la durée et le taux de renouvellement de vos contrats?

J-M.B. Ceux qui ont été renouvelés avant 1997 l'ont été pour cinq ans, ceux qui l'ont été depuis le sont pour plus longtemps. Nous avons un taux de renouvellement de $92 \%$, légèrement au-dessus de la moyenne de la profession. L'explication est simple. Dans la majorité de ses contrats, un opérateur a pour obligation contractuelle de reprendre le personnel municipal. Une façon pour les villes d'atteindre le niveau souhaité d'économie est de retirer plusieurs centaines de personnes de la liste de leurs employés municipaux. Des consultants les aident à définir les ressources qu'elles veulent transférer : des opérationnels, mais aussi beaucoup de personnes qui n'ont rien à faire. Sans le dire, les villes comptent sur nous pour faire le ménage. C'est très hypocrite parce qu'elles nous interdisent en même temps les licenciements. Il faut s'engager à reprendre 300, 400 personnes, puis procéder à des réductions d'effectifs. Nos courbes de coût ont un profil qui va en réduction au cours des trois ou quatre premières années avant d'arriver à un palier. 
Flux. La réduction d'effectifs est-elle la première source d'économie?

J-M.B. Dans l'eau, les économies proviennent du personnel et des consommations d'énergie et de produits chimiques. S'y ajoute le traitement des boues résiduaires en assainissement. L'effort est fait sur tous les postes, mais une grosse partie de l'économie vient effectivement du poste personnel. À Atlanta, il y avait 535 personnes au départ. La ville nous en a transférées 485. Depuis, on en a perdu 100 et il faut qu'on en perde encore 50, pour arriver autour de 330 . Nous avons utilisé tous les moyens disponibles qui ne sont pas des licenciements : d'abord les mises à la retraite normale, puis un test anti-drogue et un programme de retraite anticipée avec des contreparties financières. Après on fait la chasse aux économies. Pour les produits chimiques, les économies viennent d'abord de l'optimisation des process. C'est un problème de mentalité. Nous sommes une structure privée: notre finalité est capitalistique par opposition au service public qui n'a pas ce souci du «bottom line». Sa tendance serait de mettre un peu plus de produits chimiques pour ne pas prendre de risque, plus de chlore par exemple. Nous, nous faisons du pilotage, en fonction des variations de température et de consommation, et pas un traitement moyen qui satisfait toutes les conditions. Avec un pilotage en temps réel, on optimise les quantités de produits et les consommations d'énergie. Les process doivent être meilleurs et en même temps générer des économies. Dans le domaine de l'énergie par exemple, les systèmes municipaux rejettent dans l'atmosphère le méthane produit par les boues résiduaires. Nous le recyclons : la pollution disparaît et on fait une économie d'énergie. Ces améliorations supposent des investissements. Un contrat de vingt ans permet leur amortissement. Avec un contrat de trois ou de cinq ans, même si vous pouvez le renouveler, vous n'êtes pas prêts à faire des investissements significatifs, parce que vous n'avez pas le temps matériel de les amortir. Quand vous avez vingt ans, l'approche est complètement différente.

Flux. La US Environmental Protection Agency demande aux distributeurs d'adopter de nouveaux process de traitement, de réduire la consommation de chlore?

J-M.B. Plusieurs phénomènes convergent. Aux USA comme ailleurs, les normes se renforcent. Les besoins de traitement et donc les besoins d'investissement augmentent. On va produire une eau plus pure, $c^{\prime}$ est la première chose. Il faut ensuite transporter cette eau en lui gardant sa qualité. Le problème est souvent dans les réseaux. Les gens ont toujours été plus vigilants pour le traitement que pour le transport et la distribution. Une belle station, on peut la montrer, I'utiliser politiquement. Le reste est sous terre. Aux USA, une façon traditionnelle d'assurer le maintien de la qualité de l'eau en transport - distribution est de surcharger en chlore. Les Américains ont regardé ce qui se faisait ailleurs, en particulier en Europe. En changeant les produits, on améliore le goût et le comportement de l'eau. Sur un réseau qui fait $10 \mathrm{~km}$, au lieu d'avoir un point d'injection de chlore, on en a dix, pour faire un meilleur dosage. Avec des capteurs, le pilotage sera plus fin encore. C'est ça l'enjeu.

Après il y a des effets de taille. Il y a 58000 réseaux, dont 44000 desservent moins de 10000 habitants. $\mathrm{Si}$ vous imposez à ces réseaux une contrainte de traitement supplémentaire, ils auront un problème économique, un problème de taille. Il y a un besoin de concentrer.

Flux. Quelles sont, à part la durée, les principales différences entre un contrat Operation \& Maintenance (O\&M) et un contrat Operation, Maintenance \& Management (OM\&M)?

J-M.B. Dans notre jargon, on distingue les OPEX (operating expenses) et les CAPEX (capital expenses). Le contrat O\&M concerne essentiellement le management des OPEX alors que le contrat OM\&M comporte aussi la gestion des investissements. Il faut toutefois nuancer. Avec les contrats O\&M de cinq ans, UW gère sans investir. Par contre quand ils durent vingt ans, UW est amené à faire des investissements puisqu'une manière de réduire les effectifs est de mettre davantage d'automatismes, de changer des équipements anciens et coûteux en énergie. La différence est que les investissements réalisés en O\&M sont décidés par l'entreprise pour ses besoins propres.

L'implication du secteur privé est plus forte avec le management de l'investissement pour le compte de la collectivité. Nous poussons donc dans cette direction, notamment à travers la recherche de revenus supplémentaires. Prenons l'exemple de la collecte de factures. Sur tous nos services régulés, nous sommes propriétaires des actifs. Nous assumons totalement le risque clientèle : nos recettes sont les prélèvements opérés chez 
les clients individuels. Pour les contrats comme Jersey City ou Atlanta, nous sommes rémunérés pour assurer la lecture des compteurs, la facturation et la collecte des paiements, mais nous n'assumons pas le risque associé au niveau de la collecte. À Atlanta, près de $50 \%$ de l'eau distribuée n'est pas facturée à cause des pertes dans les réseaux, des compteurs défaillants ou inexistants et d'un mauvais suivi des factures. Nous avons proposé à la ville d'aller chercher ces paiements additionnels : elle nous rembourse le coût de ce service et nous partageons ensuite les recettes additionnelles. La recherche de revenus supplémentaires est un premier pas vers le CAPEX.

Flux. Atlanta est responsable de la réalisation et du financement des investissements. Avez-vous un rôle de conseil à son égard?

J-M.B. Nous avons une obligation morale sinon contractuelle de conseil. Atlanta est un bon exemple parce qu'elle n'a pas fait ce qu'elle devait faire en matière d'investissements. Cela a deux conséquences. D'abord une détérioration du service, donc un profil de risque qui change en matière de qualité de service. Pour Atlanta c'est important parce qu'elle peut être mise en défaut par l'EPA. Nous discutons de ces risques et nous arrivons souvent à modifier leur approche en terme d'investissement. L'idée est de réussir à les gérer pour eux. L'autre conséquence du manque d'investissement est qu'il entraîne des coûts additionnels en exploitation qui détériorent l'équilibre de notre contrat. C'est une discussion permanente avec Atlanta et les autres villes, c'est la vie d'un contrat. tion. Si au contraire vous réalisez des économies supérieures à celles prévues par votre business plan, la ville peut-elle renégocier sa rémunération?

J-M.B. Contractuellement non. Il y a eu un appel d'offres, un processus de sélection, une vraie compétition. Ils ont obtenu les meilleures conditions. Nous, nous avons pris un risque. Quand il y a un appel d'offres, la compétition fait qu'on accepte de prendre un certain nombre de risques. $\mathrm{Si}$ ce risque ne se matérialise pas ou si nous sommes meilleurs que prévu, notre marge augmente. C'est la règle du jeu. Mais ces risques sont encadrés par un certain nombre de conditions, notamment en terme d'investissement. $\mathrm{Si}$ la ville ne remplit pas sa part de la mission, nous ne pouvons pas remplir la nôtre ou en tout cas pas dans les mêmes conditions et il faut donc renégocier. Le système américain est un système de bonne foi. Les deux parties sont supposées être de bonne foi au moment où elles établissent leur lien contractuel. Donc si une des deux parties peut démontrer qu'elle subit un préjudice parce que l'autre n'a pas respecté sa part du marché, c'est juridiquement défendable.

Flux. Les appels d'offres sont-ils la norme?

J-M.B. Pratiquement toujours. Nous les préférons à la négociation directe qui a toujours une connotation politique et peut éventuellement être remise en cause à la suite d'un changement politique. Une procédure de mise en concurrence est beaucoup plus protectrice pour nous.

Flux. L'appel d'offres spécifie-t-il les résul-

Flux. Vos contrats sont "capés». Vous recevez une rémunération annuelle pendant 5, 10, 15 ou 20 ans en échange de services. À Atlanta \$20 millions par an pendant 20 ans. Quels sont les critères de re-négociation de ces contrats?

J-M.B. Il y en a assez peu, essentiellement les changements de lois ou de normes.

Flux. Quand vos coûts d'exploitation augmentent à cause de la détérioration des actifs, vous pouvez ouvrir une négocia- tats à atteindre ou les moyens à mettre en œuvre?

J-M.B. Il s'agit essentiellement d'une obligation de résultats. Nous prenons connaissance des paramètres d'entrée de l'eau, des paramètres de sortie en terme de distribution, et nous sommes chargés de gérer le système entre les deux.

Flux. À Indianapolis, vous avez été confrontés à une sélection un peu particulière. Vous n'étiez pas opposés à un concurrent classique mais au service d'eau de la ville? 
J-M.B. Oui, c'est la concurrence organisée. En NouvelleÉcosse, pour un gros projet de construction de réseau et de traitement des eaux usées et pluviales, Hallifax a fait organiser I'offre par ses services municipaux. Ils ont fait une proposition qui servait de comparaison aux offres privées. Si une offre privée était plus intéressante, elle était retenue mais si toutes étaient moins bonnes, les services municipaux restaient. À la Nouvelle Orléans, ils avaient imaginé la même chose. Mais la concurrence organisée était plus active il y a deux ou trois ans. Les municipalités demandent maintenant des schémas de garantie plus sophistiqués. Des sociétés comme la nôtre peuvent l'offrir parce qu'elles ont un bilan et une histoire. Les employés municipaux n'ont pas d'histoire et n'ont pas de bilan. Ils ne peuvent offrir les mêmes garanties de performance, des garanties qui, quelque part, débouchent sur des garanties financières. Avec nous il y a un vrai transfert de risque. Avec les employés municipaux, il n'y en a pas.

Flux. La résistance des employés municipaux est souvent invoquée pour expliquer la lenteur du développement des contrats de délégation?

J-M.B. C'est vrai, mais plus on s'oriente vers des choses sophistiquées qui nécessitent des moyens financiers importants, moins on aura ce problème. Les employés municipaux seront dans une situation difficile pour gérer en partenariat avec la ville des investissements. Quand il s'agira en plus de les financer ou de les co-financer, ils ne seront plus capables. Plus c'est sophistiqué et moins il y a de concurrents. Et puis nous avons une histoire suffisante : nous avons pu établir à travers les contrats qui marchent qu'il était possible de transférer des gens du municipal vers le secteur privé en maintenant des conditions égales de salaire, sans licenciement. Nous avons noué des relations de partenariat avec les principaux syndicats. Ils ont un discours officiel, mais aussi un discours beaucoup plus pragmatique quand on les rencontre à titre privé et ils sont prêts à faire des partenariats. À Indianapolis, une partie de la marge brute est reversée au personnel à travers les syndicats.

Flux. Vos services régulés sont soumis au contrôle de la Commission de régulation des services publics. Ces commis- sions doivent notamment approuver vos augmentations de tarifs. Quels sont les principes de cette régulation?

J-M.B. Cela varie un peu d'un État à l'autre, mais il y a partout une obligation de compte rendu. Il faut produire des résultats sur une base trimestrielle de façon assez légère et sur une base annuelle de façon assez complète pour chaque service, chacun ayant son tarif sur mesure. Les Commissions de régulation des services publics ont par ailleurs un droit d'audit qu'elles exercent à leur discrétion. Le mécanisme en théorie est que tous les coûts sont reconnus et que la partie du financement amenée en capitaux propres est rémunérée par le taux de retour négocié contractuellement. Ce taux est de $12 \%$ dans l'Illinois mais il varie d'un État à l'autre, en fonction de considérations fiscales. C'est nous qui sommes moteur pour aller renégocier les tarifs. Si nous sommes capables de faire mieux que ce qu'on a obtenu, parce qu'on fait des gains de productivité, la théorie est qu'on devrait le rétrocéder, la pratique c'est qu'on se tient tranquille. Par contre quand un nouvel investissement s'impose, quand il y a de l'inflation ou quand les conditions économiques ont changé, nous allons trouver le régulateur pour lui demander une augmentation des tarifs. Il faut attendre plusieurs mois, parfois jusqu'à un an et demi, avant de l'obtenir. Mais les choses évoluent. Nous avons des discussions de plus en plus ouvertes avec le régulateur à qui nous disons : votre régulation sur les fonds propres avec obligation de rétrocéder n'est pas motivante!

Flux. Comment apprécient-ils les coûts d'exploitation? S'appuient-ils sur des indicateurs, font-ils du benchmarking ?

J-M.B. Non, c'est une approche purement comptable. Ils vérifient que nous sommes capables de tout justifier. À nous d'être suffisamment professionnels pour avoir des dossiers qui tiennent la route. L'autre chose qui commence à se mettre en place : le plan d'investissements pré-approuvés sur cinq ans. II s'agit d'une augmentation de tarif préprogrammée qui correspond à des investissements préprogrammés sur cinq ans, généralement destinés à financer le renouvellement des réseaux. L'idée est de rendre le système réactif. Aujourd'hui, nous allons voir le régulateur quand nous avons besoin $\mathrm{d}^{\prime}$ investir ; puis on finit par faire l'investissement pour accélérer la procédure et 
obtenir la variation de tarif. Nous travaillons à obtenir une variation anticipée pour ne plus avoir à faire le préfinancement.

Les besoins énormes en investissement aident à ce changement. On peut jouer avec les chiffres mais quand on s'amuse à additionner tout, en terme d'eau potable, d'assainissement et d'eaux pluviales, on arrive à un chiffre qui est magique aux États-Unis, celui de $\$ 1000$ milliards. C'est le seuil à partir duquel on attire l'attention du Congrès. Le Congrès s'est saisi du dossier de l'eau il y a quelques mois parce que le seuil était atteint. Depuis, un comité est au travail. Le problème est simple : \$50 milliards d'investissement par an pendant vingt ans, sachant que les tarifs en paient $20-25$, reste un trou de \$25 à \$30 milliards par an.

Flux. L'EPA estime à $\$ 150$ milliards les investissements nécessaires à la mise en conformité du produit avec la nouvelle réglementation et au renouvellement des réseaux d'eau?

J-M.B. L'EPA a effectivement un seuil plutôt bas. Les associations professionnelles estiment que les technologies ont changé et qu'il ne suffira pas de faire de l'emplâtre. Si on considère les investissements en terme de traitement et les investissements en terme de réseaux, dont la moyenne d'âge est d'au moins cinquante ans, les besoins sont énormes. Nous arrivons donc au double du montant avancé par l'EPA. Les $\$ 1000$ milliards se décomposent en un tiers eau, un tiers assainissement et un tiers eaux pluviales.

Flux. Il semble que les Commissions de régulation des services publics s'appuient sur la satisfaction des consommateurs pour apprécier le niveau de prestation des services régulés?

J-M.B. Dans les tarifs des services régulés, une somme est ajoutée pour payer le représentant des consommateurs qui est un organisme représentatif. II a une voix sinon un vote dans les décisions qui relèvent de la qualité et de l'économie des services. À chaque fois que la Commission de régulation des services publics est sollicitée, le représentant des consommateurs accède au dossier et peut poser des questions. En théorie la Commission de régulation des services publics reste libre de son choix. En pratique, la commission de régulation et ses commissaires sont liés au pouvoir politique de l'État et il n'y a pas un politicien qui prendra le risque de mécontenter des consommateurs qui sont aussi ses électeurs. Quand on a acheté United Water Resources, il a fallu repasser devant les Commissions de régulation des services publics de tous les États où UW avait des services régulés et traiter avec les représentants des consommateurs. Dans de nombreux cas, les tarifs ont été renégociés. Face à la consolidation qui s'est produite ces dernières années, les Commissions de régulation des services publics disent: « vous nous expliquez que vous allez fusionner pour bénéficier d'économies en termes d'échelle, en termes de gestion. Mais quelle est notre participation à ces économies? » Dans le New Jersey et à New York, nous n'avons pas eu de réduction de taux, mais une période de gel de révision de nos tarifs. Pendant deux ans, nous n'avons pas le droit de demander une augmentation. D'autres opérateurs, en qui s'est produite ces dernières années, les

Commissions de régulation des services publics disent : " vous nous expliquez que vous allez fusionner pour bénéficier d'économies en termes d'échelle, en termes de gestion. Mais participation à ces économies?»
Face à la consolidation quelle est notre particulier Thames Water dans le New Jersey, ont dû rétrocéder une somme aux consommateurs à travers leurs représentants.

Flux. Avec vos partenaires contractuels, les villes, la régulation doit être assez différente : dans la mesure où vous recevez une rémunération annuelle, les villes contrôlent la qualité du produit et du service mais n'entrent pas dans le détail de vos comptes?

J-M.B. Absolument. Nous sommes rarement en situation d'affermage ou de concession. Nous n'avons donc pas la maîtrise des tarifs. Atlanta définit son tarif, c'est son problème. À Hoboken, où nous avons une concession, il y a une négociation avec la ville. Elle a ses règles propres, même si elle s'inspire largement des systèmes régulés. En particulier, cette négociation intègre le fait qu'il est raisonnable pour nous d'avoir une marge sur l'exploitation et d'avoir un retour sur investissement. Nous avons défini une formule tarifaire contractuelle qui intègre des paramètres fiscaux, l'inflation et des mécanismes liés aux besoins d'investissement et qui peuvent provoquer des renégociations tous les cinq ans. 
Flux. Quels sont vos interlocuteurs dans les villes pour négocier les contrats et ensuite pour les réguler?

J-M.B. Cela dépend de la taille de la ville et de son organisation. Mais dans une ville comme Atlanta ou Indianapolis, il y a en général un service de l'eau qui peut aussi s'appeler service des travaux publics avec à sa tête un chef de service qui fait partie de l'équipe municipale et qui a la responsabilité de l'eau. Notre interlocuteur est en général un élu local qui est nommé chef de service. Dans les grandes villes, la gestion strictement technique s'opère au jour le jour avec les services techniques de la ville. La gestion du contrat se déroule avec le chef de service et, quand il y a des évènements exceptionnels, avec quelqu'un qui est plus proche du maire, en l'occurrence à Atlanta avec le Premier Adjoint, qui fait un peu fonction de chef de cabinet. ment vis-à-vis de la ville, vis-à-vis des syndicats et vis-à-vis du personnel.

Flux. Avez-vous une approche par activité dans votre contrôle de gestion?

J-M.B. Chaque service régulé a évidement une obligation de comptabilité propre. Chaque gros contrat est organisé avec une structure ad hoc, pour des questions de responsabilité, avec une comptabilité analytique par projet. Notre système de benchmarking nous permet ensuite de comparer le coût de nos exploitations, le coût d'une opération d'un service à l'autre. Ces données alimentent notre base de données et nous servent pour de nouveaux projets.

Flux. Les commissions de régulation des services publics, en revanche, ne comparent-elles pas les opérateurs au sein d'un État puis entre États?

J-M.B. Des comparaisons sont faites par chaque commission de régulation des services publics à son échelle, mais pas entre les services relevant de différentes commissions. II faut comparer ce qui est comparable. La comparaison entre le New Jersey et la Californie, par exemple, est difficile: dans le New Jersey, les opérateurs régulés sont responsables depuis la gestion de la ressource en eau jusqu'à la distribution chez les usagers. En Californie, les entreprises de services publics ont la responsabilité de la distribution. Elles achètent l'eau potable, en sortie d'usine et quelqu'un d'autre a la responsabilité de la production. Dans le New Jersey, vous avez l'impression que ce n'est que de la forêt et quand vous passez la nuit, il y a des maisons partout. Dans une ville construite en hauteur c'est encore différent. Aux États-Unis, la plupart des grandes villes ne font pas de traitement. L'eau qui est distribuée à New York City est prélevée dans le nord de l'État. Elle est sommairement filtrée, puis injectée directement dans les réseaux, où l'on rajoute du chlore. C'est tout. La ville de New York est en défaut par rapport à l'EPA, elle paie chaque année des millions de dollars d'amende. Une ville comme Atlanta finissait par payer un peu plus de $\$ 30$ millions par an d'amende. Je ne peux pas garantir le chiffre pour New York parce que ses comptes ne sont pas faciles à lire mais ce doit être de l'ordre de $\$ 150$ millions par an. Tout dépend alors de ce que vous mettez en face. À 
New York, il a 15, 20, 25000 personnes super syndiquées et des engagements politiques pris par Monsieur Giuliani de ne pas privatiser.

Flux. S'ils voulaient vraiment investir pour améliorer la qualité de leur eau, devraient-ils déléguer la gestion des usines?

J-M.B. Absolument. Ce n'est pas la finalité des services municipaux d'amener la technologie. Dans le meilleur des cas, ils font le pilotage, l'administration et les investissements mais pas la réalisation. Un des problèmes de la ville de New York est qu'ils vont chercher l'eau très loin dans le nord de l'État. De nouveaux développements sont en cours mais les locaux disent que l'eau leur appartient et qu'ils n'ont aucune raison de la donner à New York. D'autant qu'elle est gaspillée comme ce n'est pas permis, 30 ou $40 \%$ de perte, si ce n'est pas plus. Une ville comme Montréal qui a fait l'effort de faire le diagnostic de ses réseaux est arrivée à $50 \%$ de pertes... C'est colossal. Aucune réparation n'est faite. L'eau est abondante, ils ont donc l'impression qu'elle est gratuite. Par contre New York a un problème de ressource.

Flux. Percevez-vous une différence dans vos contrats, en fonction de l'abondance ou de la rareté de la ressource? Êtes-vous incités par les commissions de régulation des services publics ou les villes à réduire la consommation d'eau et à diminuer les pertes d'eau?

J-M.B. Aux États-Unis, les consommations sont absolument énormes. Un effort commence à être fait pour réduire les quantités d'eau consommées. II s'accélère parce qu'il est aussi fait pour réduire les quantités d'énergie, en particulier en Californie. Ce sont les États plus que les municipalités qui s'en chargent. Nous menons cette politique dans nos entreprises de services publics à travers une information permanente de nos abonnés. Mais nous ne sommes pas allés jusqu'à aider au financement des changements d'équipements (chasse d'eau, lave-linge...) parce que quelque part c'est se tirer dans les pieds! Pour ce qui est des pertes, nous pouvons par exemple avoir l'obligation de faire 3000 réparations ou détections de fuite par an mais c'est plus réactif que proactif. Dans les services sous contrat, nos bénéfices sont liés à la réduction des coûts et rien d'autre. C'est la raison pour laquelle nous nous orientons vers la recherche de revenus supplémentaires: on propose aux municipalités de faire la chasse aux gaspillages d'eau, comme à Atlanta, à condition qu'elles soient motivées pour le faire. Dans les services régulés, notre marge est fonction croissante des quantités vendues. Nous ne sommes pas incités à réduire la consommation !

Flux. L'eau n'est pas chère aux États-Unis : les Américains paient les mêmes factures d'eau hors taxe et hors assainissement que les Français, en consommant deux fois plus.

J-M.B. Ils ne paient pas le prix réel. Je ne crois pas que les Américains aient des systèmes plus efficaces. Ils n'ont pas le même service, il n'y a pas les mêmes taxes, seulement quelques \%. Ils $\mathrm{n}^{\prime}$ ont pas de structure $\mathrm{d}^{\prime}$ agences de bassin. Et puis ce qu'il faut voir c'est que la valeur comptable d'un réservoir comme celui construit à Harrington Park au début du siècle est nulle. Si demain il faut faire un autre réservoir, il faudra lever tous les fonds. En France, on a généré un mécanisme qui permet de payer les investissements passés et de maintenir les systèmes. Ici, nous ne sommes pas dans une perspective de développement durable. Les Américains sont maintenant arrivés à un stade où leurs installations sont vieillissantes. Pour les remplacer et ajouter de nouvelles technologies, il va leur falloir augmenter brutalement les tarifs. Dans les services régulés, avant l'arrivée des grands groupes, il n'y avait pas non plus de provisions pour renouvellement. UW, pour ne parler que d'elle, était une société cotée en bourse. Son mode de gestion était de maintenir un certain niveau de dividende pour satisfaire ses actionnaires qui étaient de petits porteurs. UW faisait sa fierté en disant qu'elle était la société à la plus longue histoire de distribution de dividendes. Or, pour distribuer les dividendes, ils étaient arrivés à réduire les investissements. Tout est là quand on y pense. Maintenant il y a un actionnaire unique, Ondeo, qui a une approche à plus long terme. Nous avons toujours un actionnaire qui nous réclame des dividendes mais $\mathrm{C}^{\prime}$ est un autre dialogue.

Flux. Faites-vous du multi-service aux USA?

J-M.B. Non, sauf pour nos usages internes, dans le cadre de 
I'optimisation du bilan énergétique. Pour le moment Suez n'est pas entrée sur le marché de la distribution d'énergie aux ÉtatsUnis, seulement sur celui de la production. Il serait possible dans certains États d'avoir un accord multi-service avec une même collectivité, mais pas dans tous. À Indianapolis, NiSource, société d'énergie, avait racheté, en 1997, Idianapolis Water Company et une société locale de production d'énergie qui toutes deux opéraient sur le même territoire. NiSource a dû vendre la société d'eau parce que dans I'Indiana une même compagnie n'a pas le droit de servir deux utilités sur le même territoire.

Flux. Dans les États qui autorisent le multi-service, comment les commissions de régulation des services publics régulent-elles une compagnie impliquée dans plusieurs activités sur un territoire proche ou identique?

J-M.B. Les régulateurs essaient d'identifier chaque entreprise de services publics pour ce qu'elle est. Pour les services communs, ils obligent à des accords entre entreprises. Ils ne s'opposent pas à ce qu'une société régulée travaille avec ses sociétés sœurs mais ils mettent des cloisons relativement étanches. Ce type de problème existe déjà pour nous quand un de nos services régulés est voisin d'un service non régulé. Le jeu serait de faire porter tous les coûts ou le maximum des coûts sur le service régulé. Mais les régulateurs ne sont pas fous! Ils acceptent qu'il y ait des zones de recouvrement mais ils nous obligent à avoir des accords précis. Il y a toujours une marge de manœuvre mais ils l'encadrent. Légalement ils ne peuvent pas réguler le contrat. Ce qui peut arriver c'est qu'une municipalité mise en défaut par l'EPA se fasse retirer l'exploitation de son système par cette dernière qui la transfère alors à l'État. Dans ce cas, l'État utilise ses moyens de contrôle, donc sa commissions de régulation des services publics.

Flux. En terme d'organisation, faîtes-vous une différence entre vos services régulés et vos services non régulés?

J-M.B. Géographiquement, notre organisation repose sur des hubs. La gestion quotidienne des activités régulées n'est pas différente de la gestion quotidienne des activités non régulées.
Elle est donc intégrée pour tirer partie des nombreuses synergies. L'approche en terme d'investissement est très différente. Nous avons donc créé une direction technique qui a une approche globale en terme d'investissement de manière à identifier les besoins et à les programmer. Cette direction développe la planification intégrée pour nos services régulés, que I'on essaie maintenant d'étendre à nos clients municipaux. II y a donc un tronc commun, pour les opérations au jour le jour, puis des ajouts plus ou moins forts selon le type d'exploitation.

Pour nos services régulés, il existe un département entier juste pour répondre à nos obligations d'information vis-à-vis des régulateurs. Les grosses sociétés qui ont des services régulés aux États-Unis ont toutes un service central. Il faut avoir une équipe qui connaît bien les mécanismes de régulation. Ce sont des personnes qui ont une expérience plutôt financière et qui sont en contact direct avec nos différents services pour connaître leurs besoins d'investissement et améliorer le pilotage du processus de régulation.

Le marché américain est un marché très fragmenté. Une première phase s'achève avec les discussions autour du rachat de American Water Works. C'était une phase de consolidation des grosses sociétés ou de rachat des grosses sociétés par des investisseurs étrangers. La deuxième vague qui s'annonce est une vague de consolidation au niveau régional. À partir du moment où une position forte est acquise dans un État, chacun va essayer de grossir à proximité. C'est en fait une gestion du portefolio. Quelques années avant que Suez ne rachète totalement UW, nous avons vendu quatre services régulés, dont deux dans I'Illinois, à AWW. Ils n'étaient pas stratégiques pour nous. Dans le New Jersey, on fait un tiers (\$150 millions par an) de notre chiffre d'affaires de l'activité régulée. Nous avons la taille critique.

Flux. Que représente aujourd'hui votre portefeuille de services régulés par rapport aux services délégués?

J-M.B. Le régulé représente les 2/3 de notre CA, mais le non régulé est en forte croissance. En terme de population, le service rendu n'est pas exactement comparable mais sur les 7,5 mil- 
lions de personnes que nous alimentons en eau aux États-Unis, $1 / 3$ est dans les services régulés, et $2 / 3$ sont dans les non régulés. En terme d'investissement par contre, $90 \%$ de nos investissements sont réalisés dans les services régulés. Les non régulés commencent à changer même s'ils restent moins dévoreurs de capitaux que les services régulés. Pour les gros contrats comme Atlanta, il faut investir une année de chiffre d'affaires pour obtenir des économies d'échelle et être à l'optimum. Il y a une composante financière lourde dans les régulés alors que dans les non régulés nos coûts ne représentent qu'une partie des coûts du service.

Flux. Et les villes avec lesquelles vous travaillez récupèrent-elles la totalité de leurs coûts à travers leurs tarifs ?

J-M.B. Elles essaient, mais elles ont généralement accumulé un tel retard qu'elles n'en récupèrent au mieux qu'une partie. Au Canada, Toronto et Montréal se penchent sur leur comptabilité et commencent à sortir tous les coûts liés à l'eau et à l'assainissement. L'étape suivante sera de faire une régie et éventuellement ensuite de privatiser tout ou partie de ces services. New York n'a pas commencé : cette ville ne sait pas combien lui coûte son service d'eau. En fait, aux États-Unis, le meilleur et le pire cohabitent. Ce pays est très conservateur : tant qu'un système fonctionne plus ou moins, il ne change pas. Puis un jour quelqu'un fait quelque chose de différent, et si le nouveau système est intelligent, il est repris par d'autres. Une fois que le point d'inertie est passé, tout peut aller très vite. La technologie et la qualité de service sont des dimensions fondamentales et nous avons une carte importante à jouer. Ondeo dépense chaque année en R\&D plus que toute l'industrie américaine de l'eau. On peut être opposé à la globalisation mais une taille internationale donne accès à des choses qui n'existent pas au niveau local.

Flux. Ne craignez-vous pas que face aux investissements colossaux, la tendance soit à un retour des subventions dans le domaine de l'eau ici ?
J-M.B. Non, plutôt à un recul. Mais c'est effectivement un projet qui était soutenu par des sociétés d'ingénierie. Elles ont cherché à répéter ce qui s'était produit sous la précédente administration Bush, la création d'un fonds pour financer les infrastructures. Nous nous sommes complètement mobilisés contre ce projet. II n'y avait pas d'association des non régulés. Nous venons d'en créer une, qui sera officiellement annoncée le 13 septembre 2001. Elle ne regroupe que les huit plus gros, mais va s'ouvrir et nous permet de faire un lobbying efficace auprès du Congrès. Il y a une compétition forte entre ceux qui veulent et ceux qui ne veulent pas de subventions. Mais les moyens ne sont pas illimités. Aujourd'hui, il n'y a plus ou beaucoup moins d'excédent budgétaire donc, tôt ou tard, ils seront obligés d'y passer. C'est à la limite du faux problème. Nous avons fait quelques enquêtes, tout comme le Urban Water Council, organisme émanant de l'association des maires des États-Unis. Si l'augmentation des tarifs est faite intelligemment, si elle est expliquée et qu'en contrepartie les gens voient leur compte de taxes diminuer, ils seront prêts à l'accepter. Si c'est une augmentation pour une augmentation, ils ne comprendront pas. Culturellement, les Américains acceptent que des opérateurs privés puissent vivre raisonnablement de leur métier. Ils ont confiance dans leur système. À la suite de certains problèmes de contamination, les gens ont progressivement
Ce qui nous a pénalisé lors des deux dernières années, c'est la bonne santé économique. Aujourd'hui, ce qui nous aide à faire repartir le marché, et c'est intéressant à observer, c'est d'abord que l'économie a sérieusement ralenti. pris conscience du fait que l'eau ne peut pas être impunément consommée, qu'elle a un coût et qu'elle n'est pas renouvelable à $100 \%$. Le changement de culture est en train de se faire. L'enjeu du marché américain n'est ni sa taille, ni son potentiel : ils existent. C'est le temps nécessaire à leur développement. Ce sont plusieurs cycles qui se combinent: le cycle politique car c'est une décision politique et six mois avant les élections, peu de gens se décident; puis le cycle économique. Il y a dix ans, nous pensions que le moment était venu. Mais après quelques contrats, l'économie est repartie. Ce qui nous a pénalisé lors des deux dernières années, c'est la bonne santé économique. Au niveau fédéral comme au niveau des États, il y a eu du surplus. Quand il y a excédent budgétaire, certains dossiers restent fermés. 
Aujourd'hui, ce qui nous aide à faire repartir le marché, et c'est intéressant à observer, c'est d'abord que l'économie a sérieusement ralenti. Tout le monde réalise que les engagements pris ne pourront être tenus, faute de surplus. Pour prévenir les incidents ou en limiter les conséquences, les collectivités sont obligées d'aller chercher les économies où elles existent et elles se réintéressent à des projets de délégation de services. Par ailleurs, autre chose a changé, dont on ne mesure pas encore les effets. Depuis le 1er janvier 2001, les municipalités qui n'étaient pas tenues de présenter des comptes de résultat vont être obligées de le faire. Elles commencent à s'intéresser à leur endettement, à leur structure de coûts. Elles ne sont pas encore tenues de faire ressortir les charges et les produits de chaque service, et notamment de leur service d'eau, mais c'est un premier pas, une première étape. Elles voudront ensuite extérioriser une partie de leurs dettes.

Christelle Pezon Maître de conférences au CNAM (Interview réalisée à Harrington Park, au siège de United Water, le 4 septembre 2001). 